\title{
A COMPARISON THEOREM
}

\section{WALTER LEIGHTON AND WILLIAM OO KIAN KE ${ }^{1}$}

ABSTRACT. In this paper the authors consider a pair of differential equations $y_{1}^{\prime \prime}+p_{1}(x) y_{1}=0, y_{2}^{\prime \prime}+p_{2}(x) y_{2}=0$, where $p_{i}(x)$ are positive and continuous, and where solutions $y_{1}(x)$ and $y_{2}(x)$ have common consecutive zeros at $x=a$ and $x=b$. They show that if the curves $y=p_{1}(x)$ and $y=p_{2}(x)$ have a single intersection (possibly a closed subinterval) and if $p_{1}(a)>p_{2}(a), p_{2}(b)>p_{1}(b)$, the first conjugate point of $a+\epsilon(\epsilon>0$ and small) for the second equation precedes that of the first.

\section{Consider the differential equations}

$$
\begin{aligned}
& y_{1}^{\prime \prime}+p_{1}(x) y_{1}=0, \\
& y_{2}^{\prime \prime}+p_{2}(x) y_{2}=0,
\end{aligned}
$$

where the functions $p_{i}(x)$ are positive and continuous on an interval $I:[a, b+\delta](\delta>0)$. If solutions $y_{1}(x)$ and $y_{2}(x)$ of equations (1) and (2), respectively, have common consecutive zeros at $x=a$ and $x=b$, and if $p_{1}(a)>p_{2}(a)$, it follows from the Sturm comparison theorem that the curves $y=p_{1}(x)$ and $y=p_{2}(x)$ must intersect. In recent years a number of papers have been concerned with differential equations of the above type when these curves have a single point of intersection. Notable among these are Fink [2], [3], Eliason [4] and [5].

In the present paper we assume that the curves $y=p_{i}(x)$ intersect once on the interval $(a, b)$, but the intersection may be either a point or a closed subinterval of $(a, b)$. We have the following result.

THEOREM. If the curves $y=p_{1}(x)$ and $y=p_{2}(x)$ have the properties described above, if equations (1) and (2) have solutions $y_{1}(x)$ and $y_{2}(x)$, respectively, for which $x=a$ and $x=b$ are consecutive zeros, and if

$$
p_{1}(a)>p_{2}(a), \quad p_{2}(b)>p_{1}(b),
$$

then, for $\epsilon>0$ and sufficiently small, the first conjugate point of $x=a+\epsilon$ for equation (2) precedes the first conjugate point of $x=a+\epsilon$ for equation (1).

Received by the editors November 14, 1969.

AMS 1969 subject classifications. Primary 3442.

Key words and phrases. Second-order linear differential equation, conjugate point, comparison theorem.

1 This will acknowledge the partial support of the authors by the U. S. Army Research Office (Durham) under Grant numbered DA ARO D31-124-G979. 
To begin, we recall a result due to one of the present writers [6].

LeMma. If $c$ is a conjugate point of $x_{0}$ with respect to the equation ${ }^{2}$

$$
y^{\prime \prime}+p(x) y=0,
$$

then

$$
d c / d x_{0}=y^{\prime 2}\left(x_{0}\right) / y^{\prime 2}(c),
$$

where $y(x)$ is any nonnull solution of (4) such that $y\left(x_{0}\right)=y(c)=0$.

Without loss in generality, we may assume that

$$
y_{1}^{\prime}(a)=y_{2}^{\prime}(a)=1,
$$

and we first show that for $\delta$ positive and sufficiently small,

$$
y_{1}(x)<y_{2}(x) \quad(a<x \leqq a+\delta) .
$$

Note that

$$
\lim _{x \rightarrow a} \frac{y_{1}^{\prime \prime}(x)}{y_{2}^{\prime \prime}(x)}=\lim _{x \rightarrow a} \frac{p_{1}(x) y_{1}(x)}{p_{2}(x) y_{2}(x)}=\frac{p_{1}(a)}{p_{2}(a)}>1 .
$$

Since $y_{i}^{\prime \prime}(x)<0(i=1,2 ; a<x \leqq a+\delta)$, for $\delta$ small, we have $y_{1}^{\prime \prime}(x)$ $<y_{2}^{\prime \prime}(x)(a<x \leqq a+\delta)$. It follows that

$$
\int_{a}^{x} y_{1}^{\prime \prime}(x) d x<\int_{a}^{x} y_{2}^{\prime \prime}(x) d x \quad(a<x \leqq a+\delta) ;
$$

accordingly,

$$
y_{1}^{\prime}(x)<y_{2}^{\prime}(x) \quad(a<x \leqq a+\delta) .
$$

A similar argument shows that (7) implies (6).

Consider next the "wronskian"

$$
w(x)=y_{1}(x) y_{2}^{\prime}(x)-y_{2}(x) y_{1}^{\prime}(x)
$$

and its derivative

$$
w^{\prime}=\left(p_{1}-p_{2}\right) y_{1} y_{2} .
$$

Note that $w(a)=w(b)=0$ and that near $x=a, w^{\prime}>0$, while near $x=b, w^{\prime}<0$. Further, $w^{\prime}\left(x_{0}\right)=0$ for $x_{0}$ on $(a, b)$ if and only if $p_{1}\left(x_{0}\right)$ $=p_{2}\left(x_{0}\right)$. Inasmuch as the curves $y=p_{i}(x)$ have a single intersection on $(a, b)$ it follows that $w(x)>0$ on $(a, b)$. These observations lead to the conclusion that the curves $y=y_{1}(x)$ and $y=y_{2}(x)$ have no point in

2 We assume $p(x)$ to be continuous on $I$. 
common on $(a, b)$, for, if there were such a point, there would be a first such point $x=x_{1}$. The fact that $w\left(x_{1}\right)=y_{1}\left(x_{1}\right)\left[y_{2}^{\prime}\left(x_{1}\right)-y_{1}^{\prime}\left(x_{1}\right)\right]$ would then be positive would imply that $y_{2}^{\prime}\left(x_{1}\right)>y_{1}^{\prime}\left(x_{1}\right)$-which is impossible because of (6). Thus,

$$
y_{1}(x)<y_{2}(x) \quad(a<x<b) .
$$

Next, we shall show that

$$
\left|y_{1}^{\prime}(b)\right|<\left|y_{2}^{\prime}(b)\right| \text {. }
$$

Note that

$$
\frac{y_{1}^{\prime \prime}(x)}{y_{2}^{\prime \prime}(x)}=\frac{p_{1}(x) y_{1}(x)}{p_{2}(x) y_{2}(x)}<1,
$$

for $x<b$, near $b$; accordingly, $y_{1}^{\prime \prime}(x)>y_{2}^{\prime \prime}(x)$, near $b$, and

$$
\int_{x}^{b} y_{1}^{\prime \prime}(x) d x>\int_{x}^{b} y_{2}^{\prime \prime}(x) d x .
$$

It follows that

$$
y_{1}^{\prime}(b)-y_{2}^{\prime}(b)>y_{1}^{\prime}(x)-y_{2}^{\prime}(x),
$$

and an integration of (11) yields the fact that

$$
y_{1}^{\prime}(b)-y_{2}^{\prime}(b)>\frac{y_{2}(x)-y_{1}(x)}{b-x},
$$

for all $x<b$, sufficiently near $b$. Let $x$ be any fixed number near $b$, and we have $y_{1}^{\prime}(b)-y_{2}^{\prime}(b)>0$; that is, (9) holds.

The proof of the theorem may now be completed by an appeal to the lemma. For, if $c_{1}$ and $c_{2}$ are conjugate points of $x=a$ with respect to (1) and (2), respectively, we have, when $c_{i}=b$,

$$
\begin{aligned}
& d c_{1} / d a=1 / y_{1}^{\prime}{ }^{2}(b), \\
& d c_{2} / d a=1 / y_{2}^{\prime}{ }^{2}(b) .
\end{aligned}
$$

Thus, at $x=b$,

$$
d c_{1} / d a>d c_{2} / d a
$$

and the theorem is established.

\section{REFERENCES}

1. Maxime Bôcher, Leçons sur les méthodes de Sturm, Gauthier-Villars, Paris, 1917.

2. A. M. Fink, The functional $T \int_{0}^{T} R$ and the zeroes of a second order linear differential equation, J. Math. Pures Appl. (9) 45 (1966), 387-394. MR 34 \#7863. 
3. - Comparison theorems for $\int_{a}^{b} p$ with $p$ an admissible sub or superfunction, J. Differential Equations 5 (1969), 49-54. MR 38 \#1315.

4. S. B. Eliason, The integral $T \int_{-T / 2}^{T / 2} p(t) d t$ and the boundary value problem $x^{\prime \prime}$ $+p(t) x=0, x(-T / 2)=x(T / 2)=0$, J. Differential Equations 4 (1968), 646-660. MR $38 \# 1313$.

5. Walter Leighton, Some elementary Sturm theory, J. Differential Equations 4 (1969), 187-193. MR 37 \#506.

6. - Bounds for conjugate points, J. Reine Angew. Math. (to appear).

University of MisSOURI, Columbia, Missouri 65201 